\title{
Fusion following lateral mass reconstruction in the cervical spine
}

\author{
Michelle J. Clarke, MD, ${ }^{1}$ Patricia L. Zadnik, MD, ${ }^{2}$ Mari L. Groves, MD, ${ }^{2}$ Daniel M. Sciubba, MD, ${ }^{2}$ \\ Timothy F. Witham, MD, ${ }^{2}$ Ali Bydon, MD, ${ }^{2}$ Ziya L. Gokaslan, MD, ${ }^{2}$ and Jean-Paul Wolinsky, MD ${ }^{2}$ \\ 1'Department of Neurosurgery, Mayo Clinic, Rochester, Minnesota; and 'Department of Neurosurgery, Johns Hopkins University \\ School of Medicine, Baltimore, Maryland
}

\begin{abstract}
OBJECT Recently, aggressive surgical techniques and a push toward en bloc resections of certain tumors have resulted in a need for creative spinal column reconstruction. latrogenic instability following these resections requires a thoughtful approach to adequately transfer load-bearing forces from the skull and upper cervical spine to the subaxial spine.
\end{abstract}

METHODS The authors present a series of 7 cases in which lateral mass reconstruction with a cage or fibular strut graft was used to provide load-bearing support, including 1 case of bilateral cage placement.

RESULTS The authors discuss the surgical nuances of en bloc resection of high cervical tumors and explain their technique for lateral mass cage placement. Additionally, they provide their rationale for the use of these constructs throughout the craniocervical junction and subaxial spine.

CONCLUSIONS Lateral mass reconstruction provides a potential alternative or adjuvant method of restoring the loadbearing capabilities of the cervical spine.

http://thejns.org/doi/abs/10.3171/2014.10.SPINE13858

KEY WORDS en bloc resection; spinal reconstruction; lateral mass cage; cervical

$\mathrm{T}$ THE biomechanical structures of the craniocervical junction and subaxial spine transmit load-bearing forces from the skull to the torso while allowing head movement and protecting vital structures. ${ }^{31}$ At the craniocervical junction, axial forces are transmitted from the occipital condyles to the lateral masses of C-1 to the lateral masses of $\mathrm{C}-2$. This is the only portion of the spinal axis in which a central vertebral body does not provide the majority of support. ${ }^{32}$ Below C-2, the vertebral body bears the majority of the static axial load, while the lateral masses (as the only posterior elements with a degree of load-bearing capacity) bear far less load, although the proportion increases with hyperextension.?

Advances in surgical technique have allowed surgeons to perform aggressive intralesional or complete en bloc re- sections of pathological lesions throughout the spine. . $^{13,14,21}$ However, these resections often encompass large portions of the spine responsible for axial load bearing and control of lateralizing forces, resulting in gross iatrogenic instability. ${ }^{21}$ Surgeons must therefore reconstruct the spine to restore these capabilities. In some cases, anterior column reconstruction (such as strut placement following corpectomy) may not adequately replicate the natural force transmission at the craniovertebral junction, may require highly morbid surgical approaches, and may be technically demanding.

In this paper we describe 7 cases of lateral mass reconstruction, including 5 patients with cage placement and 2 patients with fibular strut graft placement. A case illustration is used to describe the general technique, and 
we discuss how this technique can be expanded for other indications. Perioperative complications and outcomes of this challenging technique are also discussed.

\section{Methods}

A retrospective review was performed of the medical records of all 7 patients who underwent lateral mass cage placement or fibular strut grafting from 2007 to 2009 at 2 academic institutions. These procedures were used as a component of cervical spine reconstruction following iatrogenic destabilization. Review of medical records was conducted in accordance with the institutional review board rules and regulations at both institutions.

Preoperative planning included CT scanning, MR imaging, staging, and biopsy where appropriate. A neoplastic process was diagnosed in all patients and included schwannoma, osteochondroma, chondrosarcoma, and chordoma. Intralesional or en bloc resection was determined on an individual basis, but it was anticipated that all patients would have gross iatrogenic instability upon the completion of the planned resection; thus, complex reconstruction was planned.

All patients underwent lateral mass cage placement or fibular graft placement as a component of spinal column reconstruction, including 1 patient who underwent placement of bilateral lateral mass cages. The docking sites of each cage are outlined in Table 1. In all cases, the lateral mass complex was removed in conjunction with tumor resection. To perform this procedure, mobilization and control of the vertebral artery was necessary. Once the resection was complete, the cage was carefully contoured to fit snugly between the highest level of resection (occipital condyle, lateral mass of $\mathrm{C}-1$, or a subaxial lateral mass) and the upper surface of the lowest remaining lateral mass. Cages were variably secured with small fragment screws (Synthes), but all cages were secured to the posterior instrumentation via a lateral mass screw placed through the cage. All cages were packed with allograft bone to ensure arthrodesis between cage and bone.

All patient notes, operative records, and imaging results were reviewed. Standard follow-up included 3-view cervical radiographs for evaluation of alignment and construct integrity at 1 month, 3 months, 6 months, 1 year, and yearly, or when patients presented with new onset pain or weakness. Pre- and postoperative CT scanning was also performed, with follow-up CT scanning performed at 6 months and then yearly as indicated. Fusion in individual patients was assessed using serial CT imaging. Absence of instrumentation failure or loosening and the incorporation of the cranial and caudal ends of the lateral mass cage was considered evidence of fusion. If a unilateral lateral mass construct was created, evidence of a contralateral fusion mass from standard arthrodesis was also necessary for evidence of fusion. Additionally, any intraoperative, perioperative, and postoperative complications were explicitly documented.

\section{Results}

Demographic data of the patients are summarized in
TABLE 1. Surgical resection and stabilization in the 5 patients who underwent cage placement

\begin{tabular}{cllc}
\hline & \multicolumn{3}{c}{ Reconstruction } \\
\cline { 2 - 4 } $\begin{array}{c}\text { Case } \\
\text { No. }\end{array}$ & Proximal Docking Site & Distal Docking Site & $\begin{array}{c}\text { Tension Band } \\
\text { Reconstruction }\end{array}$ \\
\hline 1 & Oc & C-3 & Oc-C5 \\
\hline 2 & C-1 & C-6 & Oc-T3 \\
\hline 3 & Oc & C-3 & Oc-C4 \\
\hline 4 & C-3 LM & C-5 LM & C3-5 \\
\hline 5 & bilat: occipital condyle & Left: C-4 LM; Right: & Oc-C5 \\
& & C-3 LM & \\
\hline
\end{tabular}

LM = lateral mass; Oc = occiput.

Table 2. The pre- and postoperative courses for all patients are illustrated in Fig. 1. Four men (57\%) and 3 women (43\%) with an average age of 49 years (range 16-77 years) were treated. Three patients presented with schwannoma, 2 with chordoma, 1 with chondrosarcoma, and 1 with osteochondroma. Four patients underwent intralesional tumor resection due to the extent of primary tumor (Case 5) or because preliminary pathology suggested a benign process (Cases 3, 4, and 6). Two patients underwent initial en bloc resection, ${ }^{1,2}$ and 2 patients ${ }^{6,8}$ underwent fibular strut placement for revision of instrumentation.

Postoperative CT scans obtained longer than 6 months after surgery were available for 2 patients, ${ }^{1,6}$ and there was evidence of fusion with either integration of the fibular graft (Case 6) or bridging bone with incorporation of the cage (Case 1). Patients in Cases 3, 4, and 7 had evidence of durable constructs at last follow-up; however, fusion could not be assessed due to a lack of CT imaging. One patient (Case 5) was lost to follow-up and another patient (Case 2) experienced a catastrophic hardware failure that required revision. The patient in Case 1 also experienced a hardware complication when the cage was placed extending from the occipital condyle to the vertebral body; however, when the cage was revised to bridge the lateral mass to the occipital condyle, the construct fused.

\section{Illustrative Case}

Case 2

The 60-year-old woman initially presented to an outside hospital with neck pain. She underwent MRI and CT scanning as well as biopsy sampling, which demonstrated a chondrosarcoma involving C-2, C-3, and C-4 (Fig. 2). The patient was offered a 3-stage en bloc resection, and, following a full discussion of the risks and benefits of the procedure, the patient elected to proceed.

The first stage involved placement of posterior occipitocervicothoracic instrumentation with mobilization of the tumor via a posterior approach. The patient was placed prone in a neutral position, and a standard midline approach was performed. Care was taken not to violate the tumor capsule, leaving a margin of tissue around the exophytic portion of the tumor at the C-5 and C-6 levels on the right side. Using normal anatomical landmarks, instrumentation was placed, including an occipital plate and bilateral thoracic pedicle screws from T-1 to T-5. Two rods 


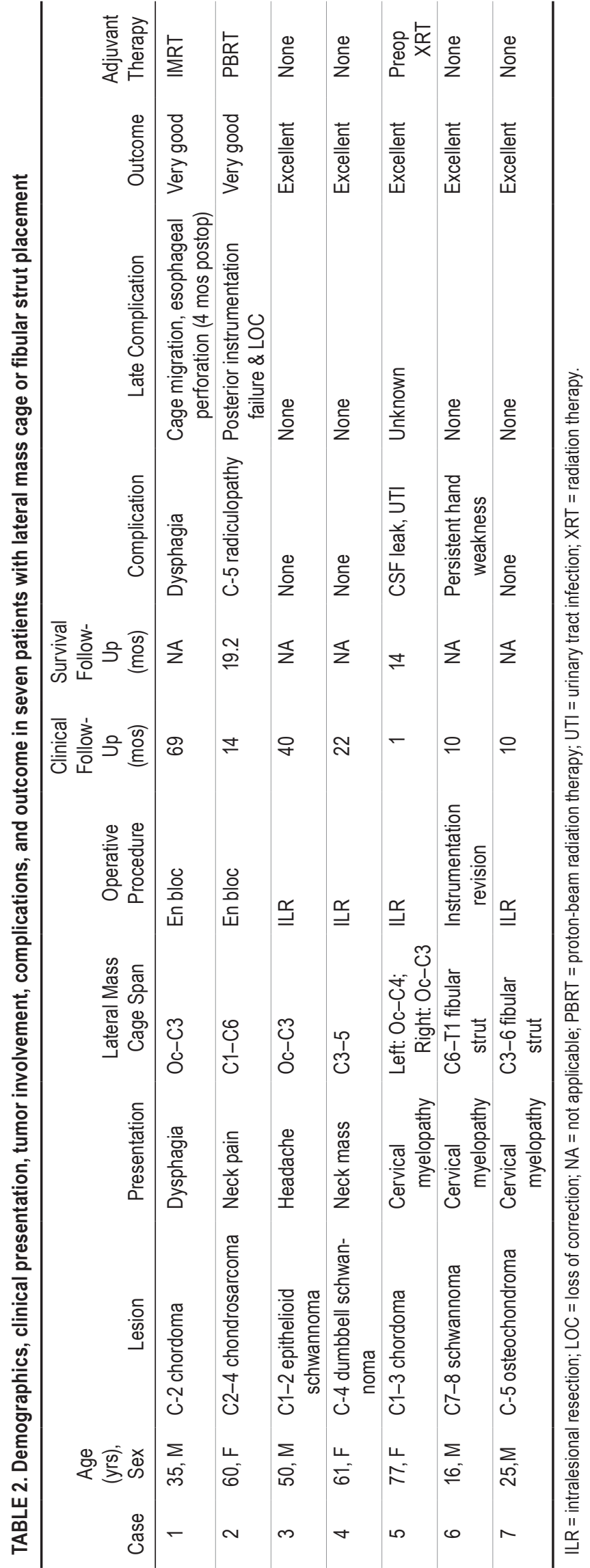

were contoured to span from the occiput to T-5 and were retained for later in the operation (Fig. 2).

Attention was turned to tumor mobilization. Laminectomies were performed at C-2, C-3, and C-4 at the junction of the lateral mass and the lamina on the left side and just approximately $0.5 \mathrm{~cm}$ off the midline on the right side, avoiding any entry into the tumor. The lateral masses of $\mathrm{C}-2, \mathrm{C}-3$, and $\mathrm{C}-4$ on the left side were resected using a high-speed diamond bur exposing the C-3 and C-4 nerve roots in their entirety. Next, the vertebral artery was mobilized. The artery was identified as it came across the C-1 arch of the sulcus arteriosus and was followed laterally to the transverse foramen of $\mathrm{C}-1$ and inferiorly to its entry into to the level of the C-4 nerve root. To mobilize the spinal segment of the vertebral artery, osteotomies were performed just medial to the vertebral artery, but through the foramen. To mobilize the rostral portion of the tumor, the arch of C-1 was removed, the C1-2 joint was identified, and the C-2 nerve roots were ligated bilaterally. This allowed further dissection ventral to the thecal sac. This approach allowed enough access to carefully cut the odontoid using a bone scalpel (Aesculap, Inc.). This allowed us to perform our rostral osteotomy through the C-2 odontoid. Achieving this osteotomy through a posterior approach was quite difficult but was attempted to eliminate the need for a transoral or transoral transmandibular approach to mobilize the rostral extent of the tumor. After this was performed, the posterior longitudinal and transverse ligaments were cut just ventral to the thecal sac. At this point of the operation, the instrumentation was assembled from C-2 through T-5 and was locked in place. The incision was closed in layers. An incidental durotomy that occurred during tumor dissection was not repaired, as better access to the ventral dura was anticipated during the third stage of the procedure. The patient was awakened, remained neurologically intact, and remained intubated overnight in the neurosurgical intensive care unit.

The second stage, completed the following day, involved an anterior approach with a C4-5 discectomy and ventral mobilization of the tumor. Prior to the procedure, a tracheostomy and lumbar drain were placed. The plastic surgery team performed a high cervical exposure via a modified carotid incision. Once exposed, identified structures included the trachea; esophagus; strap muscles; sternocleidomastoid muscle; spinal accessory nerve; internal, external, and common carotid arteries; internal jugular veins; and the C3-6 nerve roots and upper trunk of the brachial plexus. The anterior spine was exposed from the C1-2 junction to $\mathrm{C}-6$, with a good plane of tissue over the C-3 body tumor. To create the caudal extent of resection, a complete $\mathrm{C} 4-5$ anterior discectomy was performed. The posterior longitudinal ligament was incised, and a circumferential annulectomy was performed. Next, the foramen transversarium was unroofed at C-4 and C-5, allowing mobilization of the vertebral artery on the right. On the left, the vertebral artery was similarly mobilized at C-4 and C-3. A Silastic sheath was placed in the space between the trachea, esophagus, carotid sheath contents, jugular vein, cervical nerve roots, and the tumor in the spine. This was placed as a border to identify it during the Stage 3 posterior exposure. After this was completed, the area was 


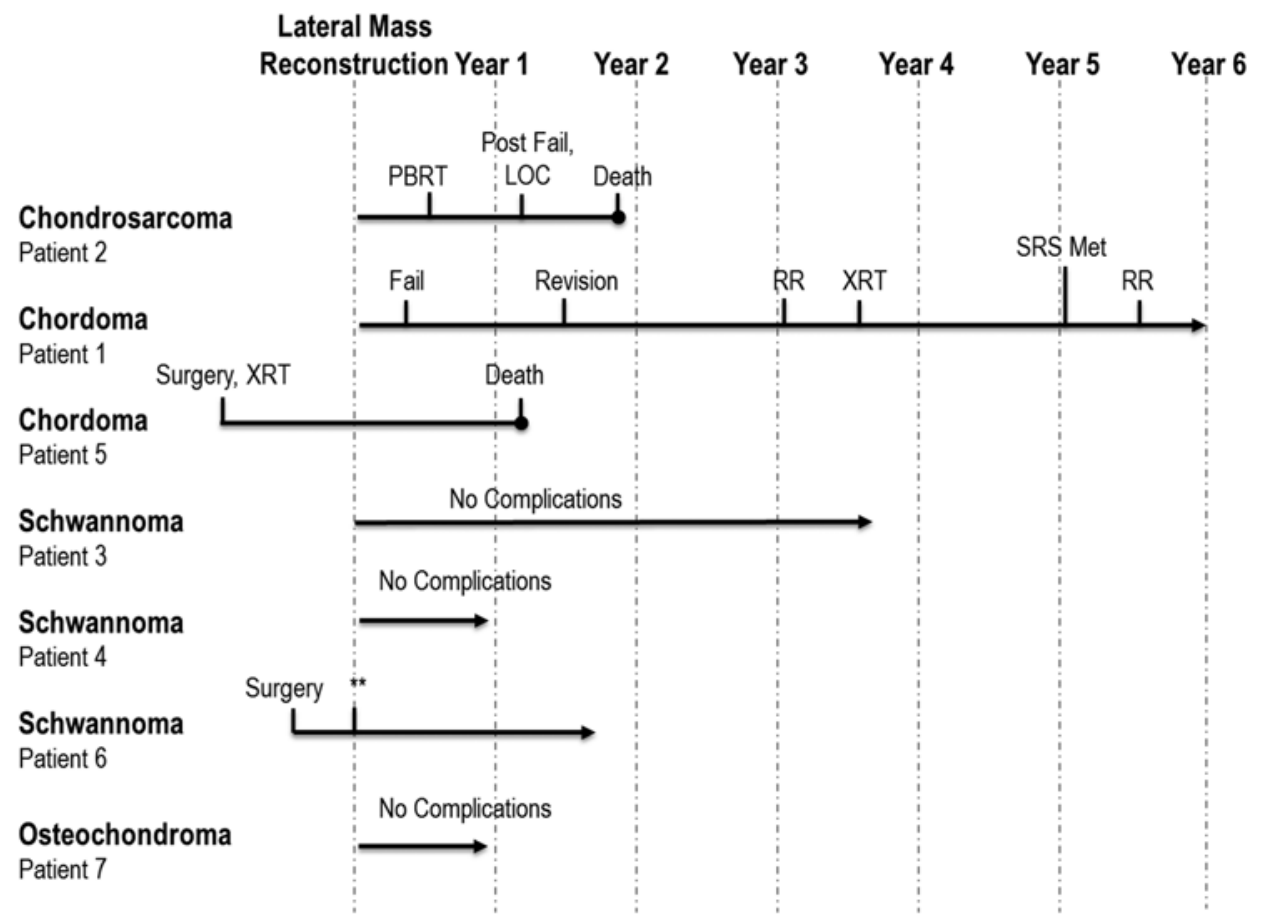

FIG. 1. Timeline illustrating postoperative course for the patients in Cases 1-7. Fail = lateral mass cage failure; LOC = loss of correction; Met = distant metastasis identified; PBRT = proton beam radiotherapy; Post Fail = failure of posterior hardware, lateral mass cage remained in place; Revision = revision of instrumentation for reason other than failure; RR = reoperation for recurrent tumor; SRS = stereotactic radiosurgery; XRT = radiation therapy. ${ }^{* *} \mathrm{~A}$ lateral mass cage was placed in the patient in Case 6 as part of a refusion procedure after posterior instrumentation failure.

then copiously irrigated with antibiotic-infused irrigation, and the incision was closed in a multilayered fashion.

Finally, a third stage encompassing tumor resection and spinal column reconstruction was completed during the same anesthesia session. The patient was positioned prone in a Mayfield headholder, and the previous posterior incision was reopened. At this point, the anterior, posterior, and caudal margins of the tumor were free. Dissection was continued laterally around the tumor until the Silastic sheath that had been placed ventrally was encountered. Dissection was completed on the right side, and the right-sided rod was removed to provide access. As the right vertebral artery passed through the tumor, it needed to be sectioned above and below the tumor. Therefore, the vertebral artery was identified at the C-1 level, mobilized to the level of C-2, ligated, and sectioned. Similarly, the vertebral artery was identified at $\mathrm{C} 4-5$, ligated, and sectioned. We cut the C-3 and C-2 nerve roots laterally. The right rod was replaced, and the left rod was removed. A similar dissection was performed on the left side. First, the previously exposed vertebral artery was further mobilized by creating osteotomies just medial to the vertebral artery at C-2, C-3, and C-4. Care was taken to preserve the C-3, C-4, and C-5 nerve roots on the left hand side.

The cranial margin was addressed next, and attention was turned to the C1-2 joint. There was still joint capsule in this region, so the joint was carefully distracted and, using sharp dissection, the capsule was cut ventrally. The rod was then replaced and locked into position. This was repeated on the contralateral side.
At this point, the mass was free of all major attachments. By pushing the mass slightly forward with the vertebral bodies of $\mathrm{C}-2, \mathrm{C}-3$, and $\mathrm{C}-4$, the plane between the tumor and the thecal sac was dissected. The right-sided C-3 and C-4 nerve roots were then cut, and the tumor was freed from the thecal sac. The vertebral bodies of $\mathrm{C}-2$, C-3, and C-4 were pushed ventrally, and all attachments between vertebral bodies and muscular attachments were cut. The specimen was delivered off to the right side out through the muscular defect that had been created. The entire en bloc specimen was passed off the field including the vertebral bodies of $\mathrm{C}-2, \mathrm{C}-3$, and $\mathrm{C}-4$, and the tumor. The previous durotomy was definitively closed primarily and covered with DuraSeal (Covidien).

Hemostasis was obtained, and attention was turned to reconstruction. Posterior instrumentation from the occiput to T-5 was in place from the first stage of the procedure. To provide load-bearing capabilities, a SynMesh cage (Synthes) to span from the right C-1 lateral mass to the right C-6 lateral mass was sized to fit. This cage was packed with demineralized bone matrix and allograft for the arthrodesis. A Synapse screw (Synthes) was driven through the cage such that the cage could be locked to the 3.5$\mathrm{mm}$ rod. The cage was placed in the position, and then 2 small fragment screws were placed locking it in position to the spine, one into the lateral mass of $\mathrm{C}-1$ and the other into the lateral mass of C-6. After the cage was positioned, both rods were replaced to span T-5 through the occiput and were locked to the cage using the Synapse screw (Fig. 2 ). The area again was copiously irrigated with antibiotic- 

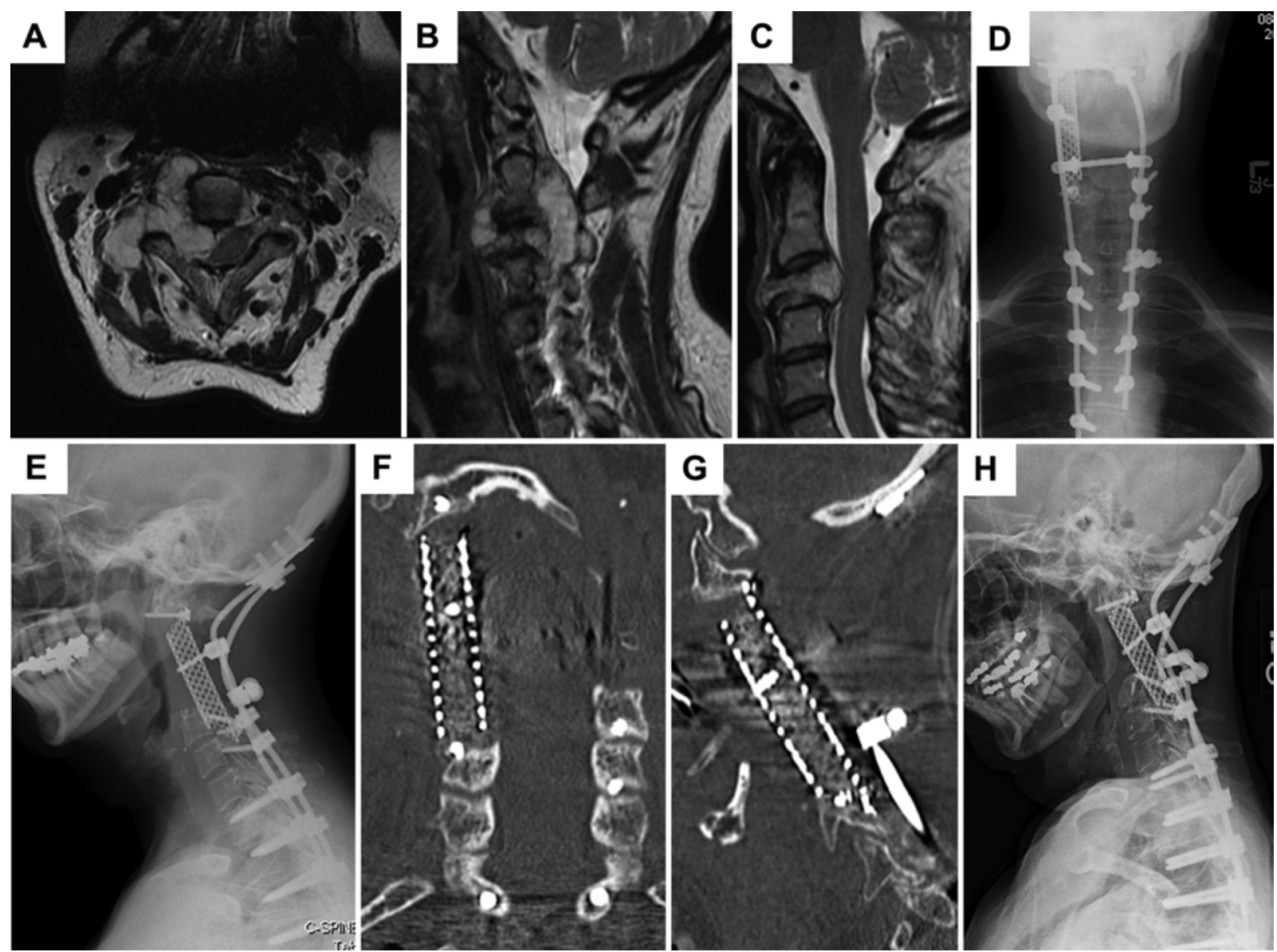

FIG. 2. Case 2. A-C: Preoperative T2-weighted MR images of biopsy-proven chondrosarcoma. A: Axial image obtained at the level of the C-4 nerve root, demonstrating the tumor originating in the C-3 vertebral body with lateral extension. B: Sagittal image obtained at the level of the lateral masses, demonstrating tumor extending from C-2 to C-4. C: midline sagittal image demonstrating C-3 pathological compression and canal compromise caused by the tumor. D-G: Postoperative anteroposterior (D) and (E) lateral radiographs demonstrating the cage extending from the lateral masses of $\mathrm{C} 1-6$. Cage placement is further visualized on coronal $(F)$ and sagittal $(G)$ CT scans. H: One year after surgery, the patient presented with neck pain and was found to have a new cervical deformity and fracture of her right posterior rod above the crossbar.

infused irrigation. The suboccipital cranium and the remaining lateral masses were decorticated, and demineralized bone matrix and allograft were packed within this region, producing arthrodesis from the occiput through T-5. At this point in the operation, the plastic surgery team was called in, and the operation completed a complex closure.

Postoperatively, the patient underwent proton-beam radiation therapy for residual disease. Fourteen months after surgery, she experienced acute neck pain and difficulty swallowing. Imaging demonstrated fracture of the posterior rods (Fig. 2), and the patient underwent reduction of her deformity with removal and reinsertion of posterior instrumentation with reinforcement of the construct (Fig. 2). She died of her disease just under 2 years after her initial resection.

\section{Chordoma Cases}

Two patients presented with chordoma (Cases 1 and 5).

\section{Case 1}

This 35-year-old man presented with difficulty swal- lowing and a tumor at $\mathrm{C}-2$ with significant prevertebral extension (Fig. 3A). He initially underwent posterior occiput to C-5 fusion, followed by en bloc resection of a C-2 chordoma. The anterior column was reconstructed using a contoured cage spanning the left occipital condyle to the C-3 vertebral body to act as a plate (Fig. 3B). This was secured with a titanium cable to the posterior fusion. Approximately 4 months after the initial surgery, the cage dislodged (Fig. 3C). The caudal end of the cage caused severe supralaryngeal region compression with imminent airway compromise and perforated the esophagus resulting in regional infection. The cage was removed and replaced with a cage spanning from the left occipital condyle to the superior portion of the C-3 lateral mass (Fig. $3 \mathrm{C}$ and $\mathrm{D}$ ). The caudal end of the cage was cut a little bit taller dorsally to also function as a plate that could be screwed into the lateral mass of C-3. One and a half years after the initial surgery, the patient underwent mobilization of $\mathrm{C} 1-2$ to provide greater range of motion to facilitate swallowing. Three years after his original resection, the chordoma recurred at $\mathrm{C} 4-5$ (Fig. 3E). The patient 

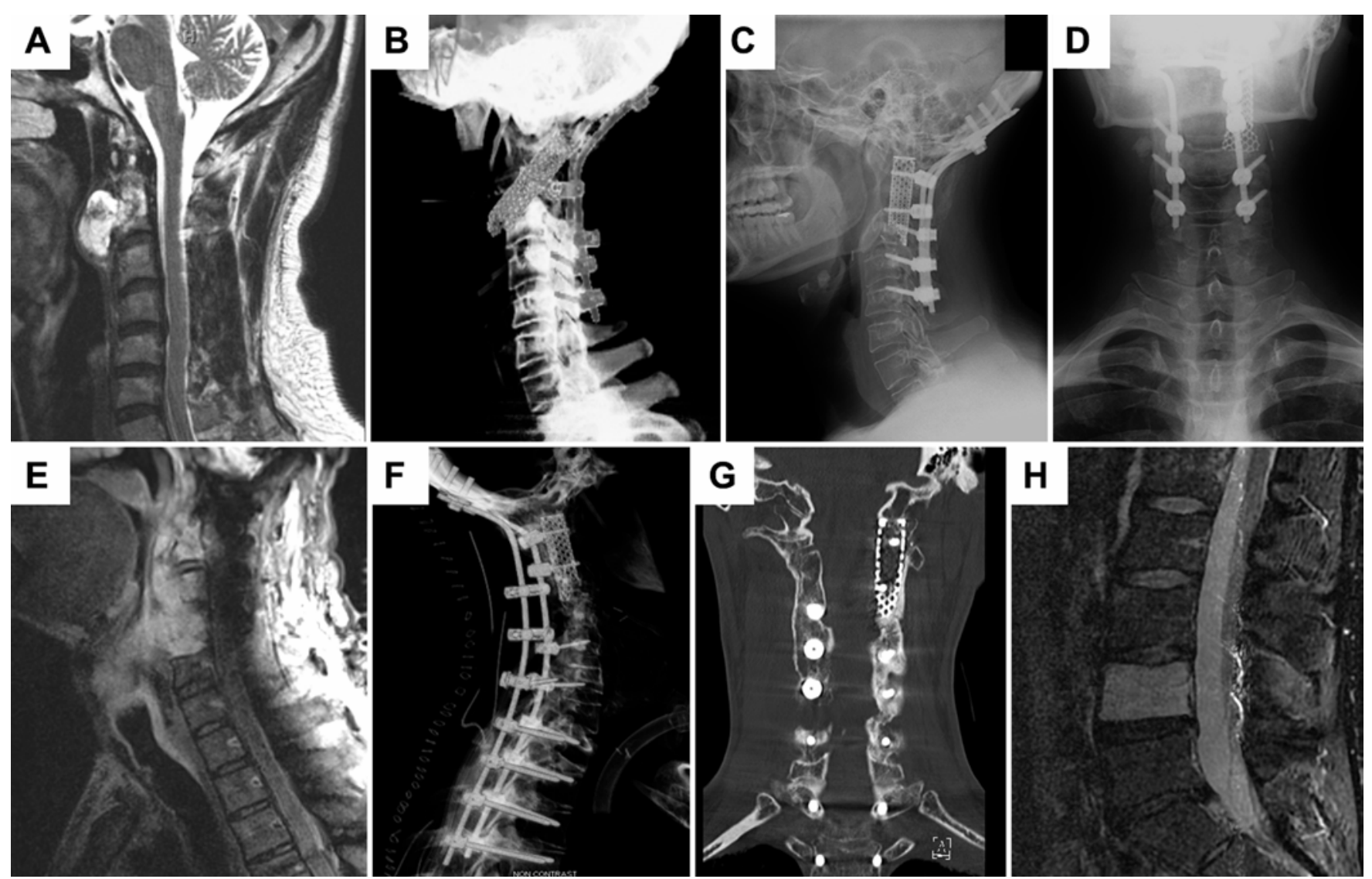

FIG. 3. Case 1. This patient presented with pain and difficulty swallowing. A: Preoperative sagittal fat-suppressed T2-weighted MRI demonstrating tumor in the C-2 vertebral body with a soft-tissue component obstructing the oropharynx. B: On 3D CT reconstruction, the cage extending from the left occipital condyle to the C-3 vertebral body has a more oblique position and has slipped further anterior to the C-3 vertebral body projecting into the prevertebral soft tissues and bulging into the posterior oropharynx. C and D: Following surgery to correct the cage migration, postoperative lateral $(C)$ and anteroposterior $(D)$ radiographs demonstrate occipitocervical fusion to C-5 with lateral mass screws from C-3 to C-5. The cage is seen extending from the left occipital condyle to the superior aspect of C-3. E: Fat-suppressed sagittal T2-weighted MR image demonstrating recurrent chordoma extending to the C4-5 disc space. F and G: Reconstructed CT scans (sagittal, F) showing extension of the posterior fusion construct to T-4 after resection of recurrent chordoma with evidence of fusion on the coronal image (G). H: Sagittal fat-suppressed T2-weighted MR image showing metastatic lumbar chordoma in this patient can be seen at L-4.

underwent a repeat anterior cervical approach with C5-6 discectomy, C4-5 corpectomy, and resection of recurrent chordoma. Occipitocervicothoracic instrumentation was extended through T-4 (Fig. 3F). Postoperatively the patient received image-guided intensity-modulated radiation therapy (IMRT) that was complicated by esophageal stricture and multiple dilations; however, bridging bone was noted on follow-up CT imaging 4 years after his initial surgery, with bony integration of the construct (Fig. 3G). He later developed an L-4 metastasis and received radiosurgery at this level (Fig. $3 \mathrm{H})$.

\section{Case 5}

This 77-year-old woman presented with cervical myelopathy and a history of surgery for cervical chordoma and radiation therapy to the region. A significant prevertebral component was noted on CT scanning (Fig. 4A) as well as complete replacement of the C-2 lateral masses by tumor (Fig. 4B). The patient was taken to the operating room for removal of posterior hardware and reconstruc- tion of bilateral lateral masses using titanium cages spanning from the occiput to C-3 (left) and C-4 (right) (Fig. 4C and D). This patient was lost to follow-up; however, she died 14 months after placement of the bilateral lateral mass cages.

\section{Other Cases}

The patients in Cases 3, 4, and 6 underwent surgery for schwannoma and experienced no postoperative complications. The patients in Cases 6 and 7 underwent fibular strut reconstruction.

\section{Case 3}

This 50-year-old man presented with headache and was found to have a C1-2 schwannoma (Fig. 5A and B). The tumor was resected in an intralesional fashion with placement of a mesh cage spanning from the occipital condyle to the lateral mass of $\mathrm{C}-3$ (Fig. $5 \mathrm{C}-\mathrm{H}$ ). No postoperative CT scans were available at 6 months or 1 year after surgery to conclude if a fusion mass was present; however, at 

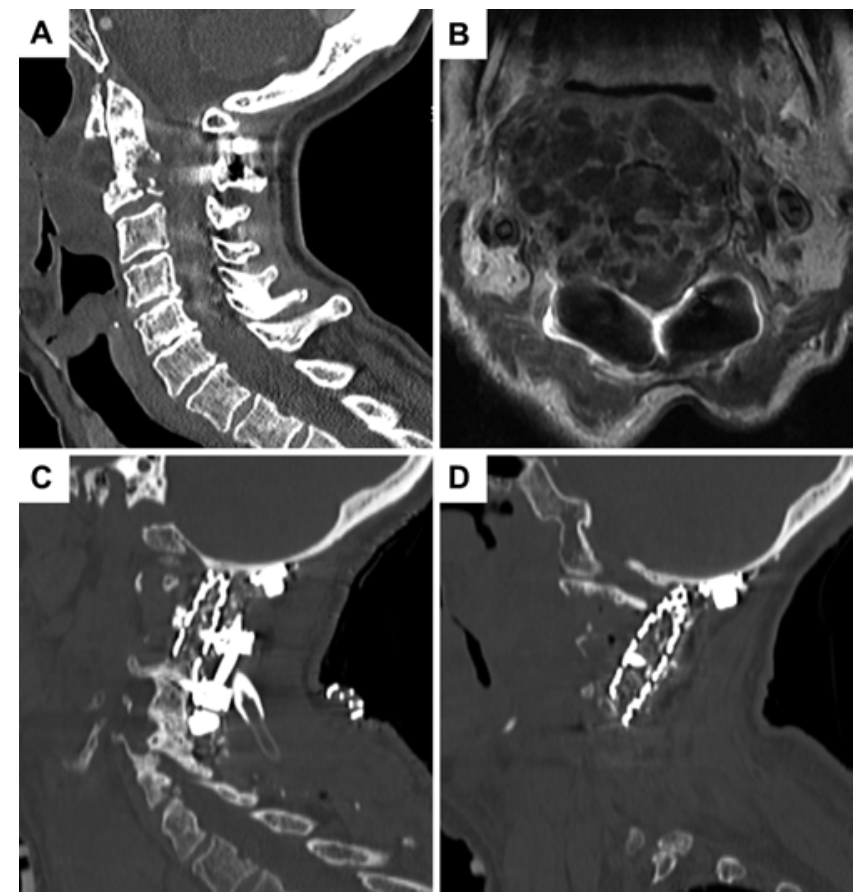

FIG. 4. Case 5. This patient presented with C1-3 chordoma and previous $\mathrm{C} 1-5$ instrumented fusion with biopsy, followed by radiotherapy. A: CT scan showing progression of the patient's disease and prevertebral growth of her tumor. B: Axial preoperative T1-weighted MR image with contrast showing large tumor at $\mathrm{C}$-2 with heterogeneous enhancement and metallic artifact posteriorly. C and D: Postoperative CT scans showing bilateral lateral mass reconstruction.

3 years after surgery, the construct remains in place (Fig. $5 \mathrm{G}$ and $\mathrm{H}$ ).

\section{Case 4}

This 61-year-old woman presented with a neck mass and was found to have a C-4 dumbbell-shaped schwannoma. This was resected in an intralesional fashion with lateral mass cage placement from C-3 to C-5, with the superior cage hooked over the posterior part of the superior lateral mass landing zone (Fig. 6).

\section{Case 6}

This 16-year-old boy presented with hand weakness and numbness, and was found to have a schwannoma at C-7 with extension through the expanded right C-8 neuroforamen. This was resected initially via laminectomy at C6-T1 and facetectomy of C7-T1, followed by posterior arthrodesis from C-5 to T-2. Six months later, the patient developed new hand weakness and evidence of loosening screws and loss of the cap of the uppermost screw. He returned to the operating room for removal of the posterior hardware, placement of a fibular strut from C-6 to T-1, and replacement of posterior instrumentation. Six months after surgery, there was evidence of solid fibular graft fusion (Fig. 7).

\section{Case 7}

Similar to Case 6, this patient underwent fibular strut reconstruction. A 25-year-old man presented with weakness in his triceps, biceps, and deltoid muscles. Imaging revealed an osseous lesion extending from the C-5 vertebral body and extending to the foramina (Fig. 8A-C). He underwent resection of his tumor and reconstruction of the C4-5 lateral masses with a fibular strut extending from C-3 to C-6 (Fig. 8D). Ten months after surgery, radiographs demonstrated construct integrity (Fig. 8E and F). An artist's rendition of a hypothetical construct is illustrated in Fig. 9 for clarity.

\section{Postoperative Course}

Of note, patients with benign pathologies had better outcomes, both in terms of survival and maintenance of correction. The patients in Cases 3, 4, 6, and 7 underwent surgery for reconstruction following resection of a benign pathology (schwannoma or osteochondroma). None of these patients experienced construct failure or loss of correction when the cage or fibular graft was placed extending from the lateral mass to an adjacent lateral mass or occipital condyle. Their postoperative courses were similarly uncomplicated by infection or other issues.

Of the 7 patients, 2 patients with malignant pathologies (chordoma and chondrosarcoma) had postoperative instrumentation-related complications. Following surgery, both patients were referred to the radiation oncology department for consideration of adjuvant therapy; 1 patient (Case 2) ultimately underwent proton-beam radiation therapy and the other patient (Case 1) underwent IMRT when the tumor recurred. The latter patient experienced catastrophic hardware failure following $\mathrm{C}-2$ chordoma resection and placement of a cage spanning from the occipital condyle to the $\mathrm{C}-3$ vertebral body. After revision of this construct to span from the condyle to the C-3 lateral mass, there was no loss of correction or instrumentation failure. The other patient also experienced a construct failure with loss of correction due to posterior rod fracture. Minor complications included a CSF leak and urinary tract infection (Case 5), and persistent hand weakness (Case 6) (Table 2).

\section{Technical Notes}

While each case has unique aspects such as patient age and exact location of the reconstruction, the general concept of spanning a gap terminating in a lateral mass is consistent. In all cases, both the cranial and caudal "landing zones" must be competent to support the cage, and time must be spent ensuring that the cage fits tightly. Additionally, as the caudal facet has a profound downward angle, the surgeon must realize that the inferior corner of the cage will tend to kick backward under axial load. Thus, we advocate placing a screw directly into the cage/lateral mass landing zone and a second screw through the cage attached to the rod to provide dorsal support and counteract this tendency. Similarly, the cranial landing zone must be considered. While the occipital condyles and lateral mass of $\mathrm{C}-1$ are relatively flat, the underside of the lateral masses of the subaxial spine will tend to cause the cage to fail by pushing the anterior aspect forward. Thus it is wise for the surgeon to "hook" the back of the cage over the posterior corner of the superior facet (Fig. 6). 

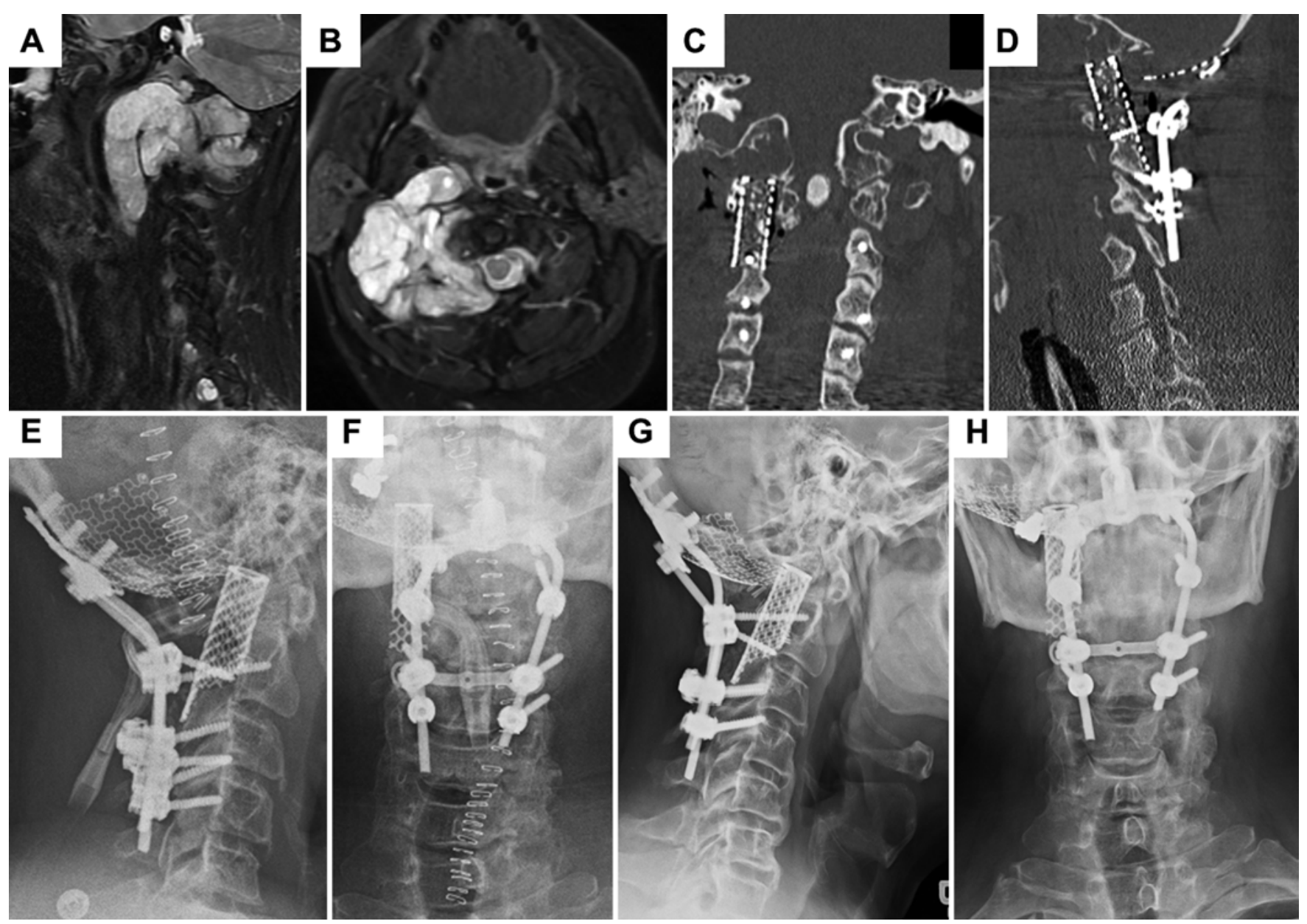

FIG. 5. Case 3. Preoperative fat-suppressed T2-weighted sagittal (A) and axial (B) MR images demonstrating lobular hyperintense mass at the skull base. Postoperative coronal (C) and sagittal (D) CT scans illustrating cage extending from the right occipital condyle to the $\mathrm{C}-3$ lateral mass. Lateral $(\mathrm{E})$ and anteroposterior $(\mathrm{F})$ radiographs obtained immediately postoperatively. Lateral (G) and anteroposterior $(\mathrm{H})$ radiographs obtained 3 years after surgery, showing maintenance of construct integrity.

The patients in Cases 6 and 7 underwent reconstruction with a cadaver fibula using the same technique discussed in Case 2 (Illustrative Case). Postoperative CT scanning at 6 months demonstrated excellent fusion with incorporation of the graft into the superior and inferior facets in one patient (Case 6), and the other patient (Case 7) had no evidence of instrumentation migration or failure at 10 months after surgery. Although it is generally considered erroneous to place a screw through a bone graft as this may create a stress riser, this was done to create an anteriorly directed force to push the fibular strut into the facets. In this case, pilot holes were carefully drilled and tapped through the strut, prior to the placement of both screws, and the graft/screw construct was installed as a single unit.

\section{Discussion}

Recently, advances in surgical techniques ${ }^{16,17,28,29}$ have allowed surgeons to aggressively resect tumors in the cervical spine; however, the resulting iatrogenic instability has been difficult to address. Traditionally, major intralesional resection was the procedure of choice in patients with primary bone tumors such as chordoma ${ }^{4}$ However, a more aggressive en bloc resection offers a survival benefit over intralesional resection. ${ }^{6,13,25,33}$ However, some authors have noted that the complexity of cervical spine anatomy may make en bloc resections of many tumors impossible. ${ }^{3}$ At this point, en bloc resection of cervical lesions is limited to case reports and small series. . $^{2,8,9,11,13,18}$

Reconstruction of the load-bearing capacity of the upper cervical spine following large resections is challenging. ${ }^{20}$ In many cases, definitive resection of pathological processes is avoided due to the risk of iatrogenic instability, and patients simply experience stabilization posteriorly. ${ }^{10,19}$ Previously, if resection was attempted and the major load-bearing structures were removed (including the occipital condyles, $\mathrm{C}-1$ lateral masses, and the C-2 vertebral body), posterior fixation was extended, usually from the occiput to the subaxial or thoracic spine. However, this alone severely compromises the anterior support. Multiple creative anterior reconstruction techniques have been described, all limited to case reports.

In cases in which the axis is resected but the atlas and occipital condyles are preserved, a C-2 prosthesis has been described that transmits forces from the C-1 lateral masses to the vertebral body of the highest unresected 

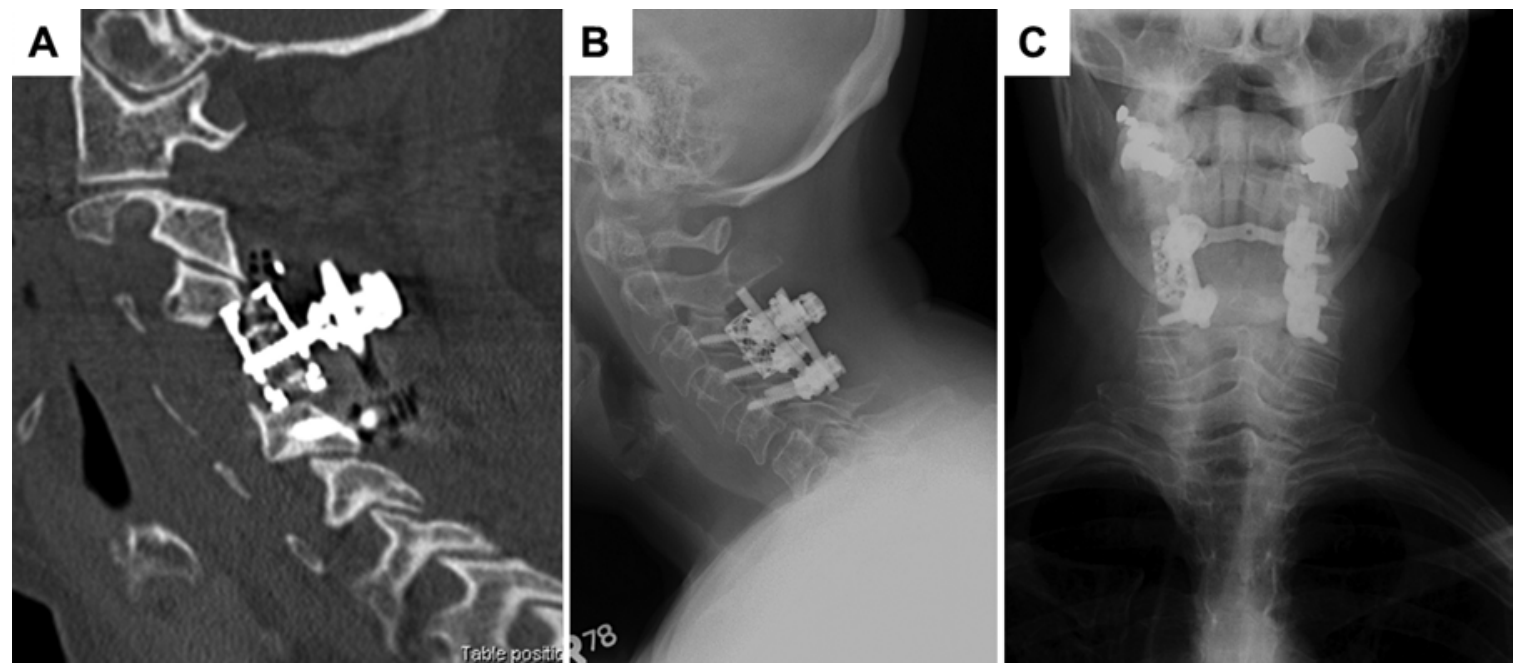

FIG. 6. Case 4. A: Postoperative CT scan. Note superior cage hooked over the posterior part of the superior lateral mass landing zone. B and C: Lateral (B) and anteroposterior (C) radiographs showing instrumentation at 6 months postoperatively.

level. ${ }^{15,22}$ The device is a modified cylindrical cage; however, instead of the cylinder supporting an upper vertebral body, the C-1 lateral masses sit on mesh struts extending laterally. To secure it, a plate connects the device to the anterior arch of $\mathrm{C}-1$. The advantage of this device is that it replicates physiological transmission of forces through the C1-2 joints and the subaxial anterior column. Additionally, the construct allows continued occipitocervical movement through occipitocervical articulation. However, the use of this device requires an intact atlas, which was only present in one of our high cervical cases. Additionally, the procedure requires a high anterior cervical approach with transpharyngeal placement of the anchoring screws at $\mathrm{C}-1$. Similar cage and plate constructs have also been described. ${ }^{26}$

Lateral mass cages provide an alternative spinal column reconstruction method, allowing maintenance of load-bearing capacity in the face of major resection. Wang et al. described the use of a lateral mass cage following resection of the C-1 lateral mass. ${ }^{30}$ In that case, the cage spanned the occiput to C-2 lateral mass and was secured with a screw through the cage into the occipital condyle. The patient underwent occiput to C-4 fusion in the same setting. Effectively, this restored normal force transfer from occipital condyle to the $\mathrm{C}-2$ lateral mass via cage instead of the C-1 lateral mass. Similar approaches have been used in the past with bone grafts. ${ }^{5,12}$ Another surgical team reconstructed a unilateral $\mathrm{C}-1$ lateral mass excision with a similar occipital condyle to C-2 body using a Harms cage via combined direct lateral and posterior approach.

This technique has also been used in conjunction with a cage spanning the clivus to $\mathrm{C}-3$ in an attempt to provide 3 -column reconstruction following intralesional chordoma resection. ${ }^{27}$ Following en bloc chordoma resection resulting in 3-column disruption, the authors reconstructed the anterior column with a mesh cage spanning the clivus to $\mathrm{C}-3$, and lateral mass cages spanning $\mathrm{C}-1$ to $\mathrm{C}-3$ and C-1 to C-4, supplemented with posterior instrumentation for tension banding. The authors felt that this construct would increase "surface area of potential bone fusion" and "offload the strain on the posterior occipitocervical fixation." This was done as a previously described case of an anterior strut from C-1 to C-5 without lateral mass augmentation failed, ultimately requiring a transmandibular, circumglossal approach and complex reconstruction that was associated with high morbidity. ${ }^{23}$ However, the premise of adding lateral mass cages to an anterior reconstruction following C-2 spondylectomy to increase stability was recently disproven in laboratory biomechanical testing. ${ }^{24}$ No biomechanical testing of lateral mass cages without anterior column support has been performed.

It is important to note the failure pattern mentioned in this series. Of the 7 patients, only the 2 patients who re-
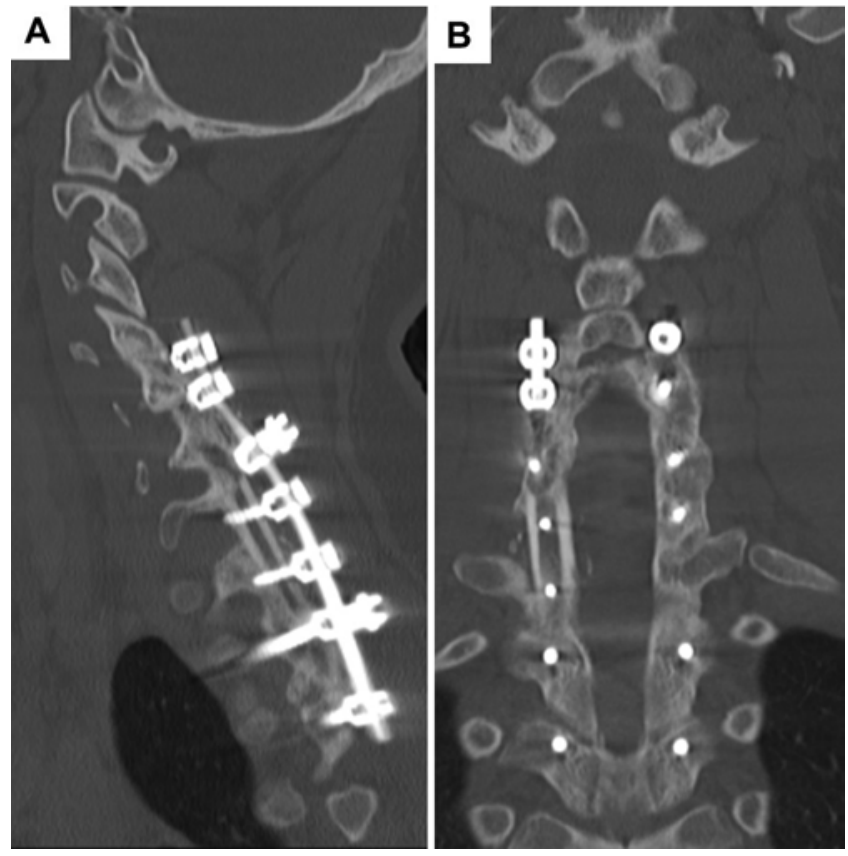

FIG. 7. Case 6. Sagittal (A) and coronal (B) postoperative CT scans obtained at 6 months, showing complete integration of fibular strut. 

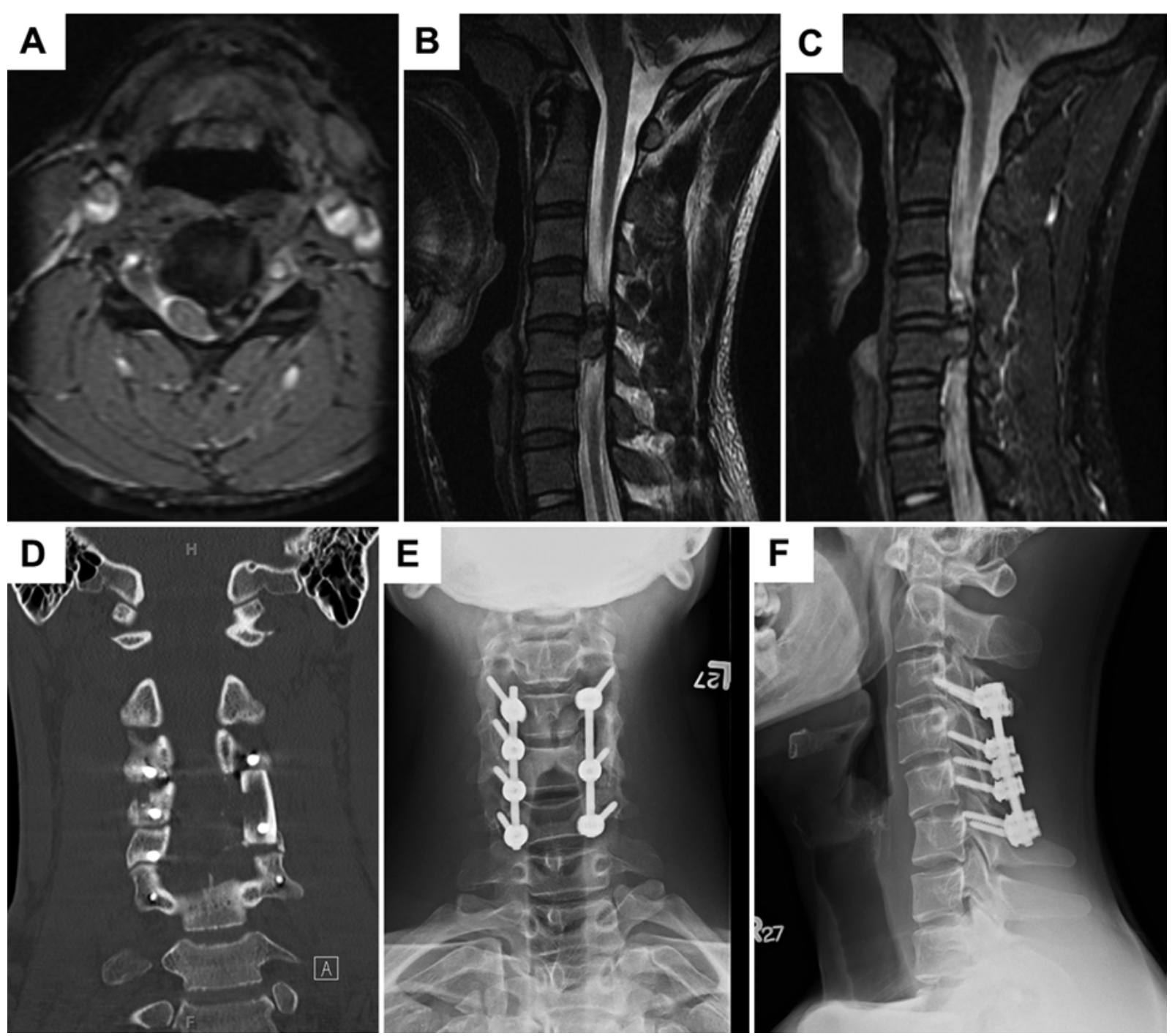

FIG. 8. Case 7. A-C: Axial (A) and sagittal (B) T2-weighted and fat-suppressed sagittal (C) MR images demonstrating an osseous exophytic lesion arising from the posterior aspect of the $\mathrm{C}-5$ vertebral body. The lesion extends superiorly to the $\mathrm{C} 4-5$ foramina. D: Postoperative CT scan showing bone graft reconstruction of the C4-5 lateral mass with fibular strut. E and F: Anteroposterior $(\mathrm{E})$ and lateral $(\mathrm{F})$ radiographs obtained 10 months postoperatively, demonstrating construct integrity.

ceived radiation treatment shortly after their surgery suffered lateral mass reconstruction failure. The remaining patients who did not undergo radiation therapy demonstrated documented fusions. It is likely that the patients who received radiation therapy never achieved a solid fusion due to the cytotoxic nature of their therapy, unlike the remaining patients. Thus, this construct type may not be appropriate for patients expected to withstand fixationonly reconstruction without true graft/cage incorporation. Extrapolating from this information, close follow-up in all patients who have not achieved solid fusion is wise.

In all cases described, the lateral mass cage span was dictated by the extent of tumor resection and subsequent reconstruction needs with the cage contoured to fit the space between the remaining levels. In most cases we have chosen to use a titanium mesh cage for reconstruction, although other options such as allograft or autograft bone may be viable alternatives. Of note, some surgeons may consider a vascularized autograft bone, especially in cases in which postoperative radiation is anticipated, to increase the chances of bony fusion. ${ }^{1}$

\section{Conclusions}

Lateral mass reconstruction may be beneficial in cases of complex reconstruction of the cervical spine, and a fibular graft or titanium mesh cage can be used. Data from this cohort suggest that reconstruction should involve posterior instrumentation for additional stabilization and screws placed directly into the cage or graft. Furthermore, the cage or graft should extend from the occipital condyle or lateral mass directly to another lateral mass, as instrumentation failure was noted when the cage extended from the occipital condyle to the vertebral body. This case cohort includes a variety of benign and malignant pathologies and includes patients who underwent adjuvant radiotherapy. 

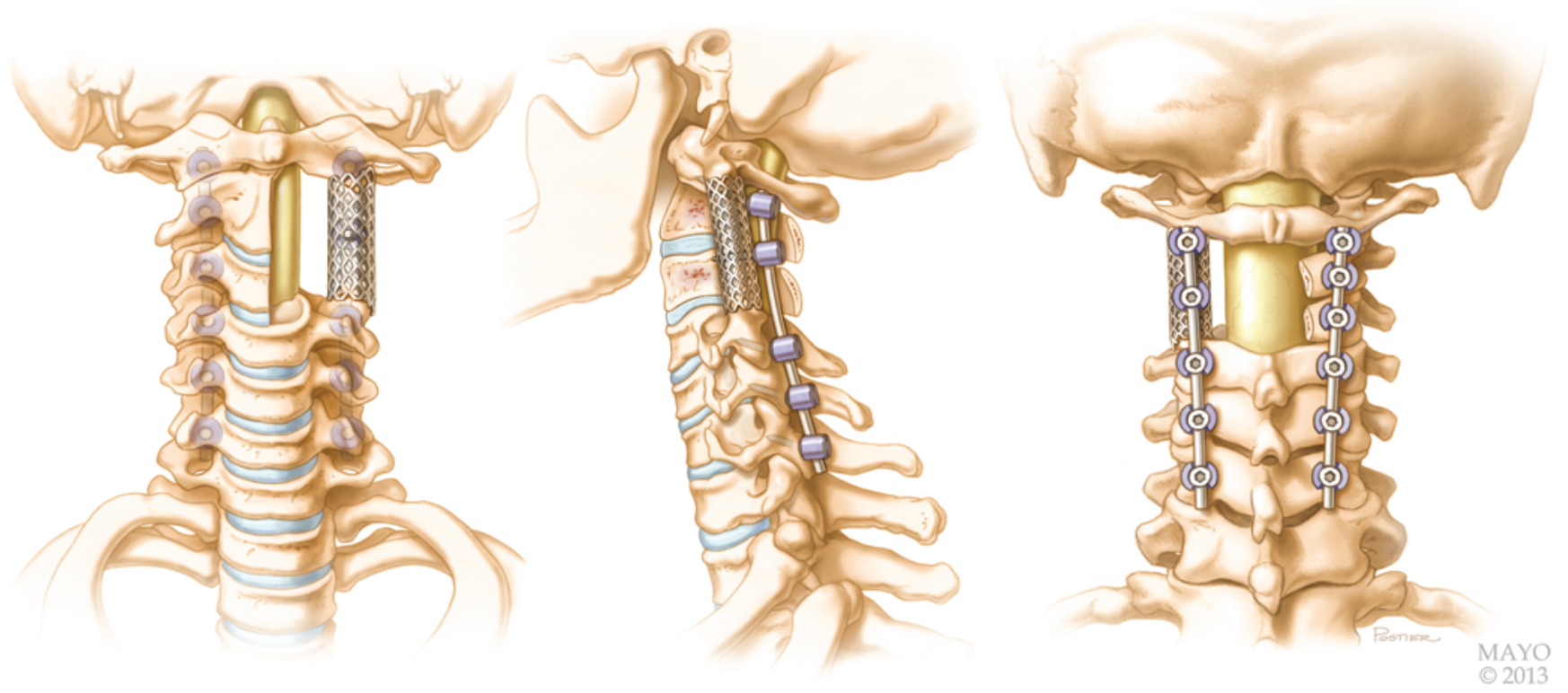

FIG. 9. Artist's rendition of a hypothetical lateral mass cage construct. By permission of Mayo Foundation for Medical Education and Research. All rights reserved.

Fusion was noted in 2 patients, and durable maintenance of correction was noted in 3 additional patients, strongly suggesting that lateral mass reconstruction with a cage or fibular graft can result in stable long-term correction for oncology patients. Surgeons should follow these patients closely during the healing process and beyond, as catastrophic failure of instrumentation was noted in 1 patient after lateral mass reconstruction.

\section{References}

1. Ackerman DB, Rose PS, Moran SL, Dekutoski MB, Bishop AT, Shin AY: The results of vascularized-free fibular grafts in complex spinal reconstruction. J Spinal Disord Tech 24:170-176, 2011

2. Bailey CS, Fisher CG, Boyd MC, Dvorak MF: En bloc marginal excision of a multilevel cervical chordoma. Case report. J Neurosurg Spine 4:409-414, 2006

3. Barrenechea IJ, Perin NI, Triana A, Lesser J, Costantino P, Sen C: Surgical management of chordomas of the cervical spine. J Neurosurg Spine 6:398-406, 2007

4. Bohlman HH, Sachs BL, Carter JR, Riley L, Robinson RA: Primary neoplasms of the cervical spine. Diagnosis and treatment of twenty-three patients. J Bone Joint Surg Am 68:483-494, 1986

5. Bongioanni F, Assadurian E, Polivka M, George B: Aneurysmal bone cyst of the atlas: operative removal through an anterolateral approach. A case report. J Bone Joint Surg Am 78:1574-1577, 1996

6. Choi D, Melcher R, Harms J, Crockard A: Outcome of 132 operations in 97 patients with chordomas of the craniocervical junction and upper cervical spine. Neurosurgery 66:5965,2010

7. Chung JY, Kim JD, Park GH, Jung ST, Lee KB: Occipitocervical reconstruction through direct lateral and posterior approach or the treatment of primary osteosarcoma in the atlas: a case report. Spine (Phila Pa 1976)37:E126-E132, 2012

8. Cloyd JM, Chou D, Deviren V, Ames CP: En bloc resection of primary tumors of the cervical spine: report of two cases and systematic review of the literature. Spine J 9:928-935, 2009

9. Currier BL, Papagelopoulos PJ, Krauss WE, Unni KK, Yaszemski MJ: Total en bloc spondylectomy of C5 vertebra for chordoma. Spine (Phila Pa 1976) 32:E294-E299, 2007

10. Fourney DR, York JE, Cohen ZR, Suki D, Rhines LD, Gokaslan ZL: Management of atlantoaxial metastases with posterior occipitocervical stabilization. J Neurosurg 98 (2 Suppl):165-170, 2003

11. Fujita T, Kawahara N, Matsumoto T, Tomita K: Chordoma in the cervical spine managed with en bloc excision. Spine (Phila Pa 1976) 24:1848-1851, 1999

12. George B, Archilli M, Cornelius JF: Bone tumors at the cranio-cervical junction. Surgical management and results from a series of 41 cases. Acta Neurochir (Wien) 148:741-749, 2006

13. Hsieh PC, Galia GL, Sciubba DM, Bydon A, Marco RA, Rhines L, et al: En-bloc excision of chordomas in the cervical spine: review of five consecutive cases with over 4-year follow-up. Spine (Phila Pa 1976) 36:E1581-E1587, 2011

14. Hsu W, Wolinsky JP, Gokaslan ZL, Sciubba DM: Transoral approaches to the cervical spine. Neurosurgery $66(3$ Suppl):119-125, 2010

15. Jeszenszky D, Fekete TF, Melcher R, Harms J: C2 prosthesis: anterior upper cervical fixation device to reconstruct the second cervical vertebra. Eur Spine J 16:1695-1700, 2007

16. Kawahara N, Tomita K, Matsumoto T, Fujita T: Total en bloc spondylectomy for primary malignant vertebral tumors. Chir Organi Mov 83:73-86, 1998

17. Kawahara N, Tomita K, Murakami H, Demura S: Total en bloc spondylectomy for spinal tumors: surgical techniques and related basic background. Orthop Clin North Am 40:47-63, vi, 2009

18. Leitner Y, Shabat S, Boriani L, Boriani S: En bloc resection of a C4 chordoma: surgical technique. Eur Spine J 16:22382242, 2007

19. Mazel C, Hoffmann E, Antonietti P, Grunenwald D, Henry M, Williams J: Posterior cervicothoracic instrumentation in spine tumors. Spine (Phila Pa 1976) 29:1246-1253, 2004

20. McLoughlin GS, Sciubba DM, Suk I, Bydon A, Witham T, Wolinsky JP, et al: Resection of a retropharyngeal craniovertebral junction chordoma through a posterior cervical approach. J Spinal Disord Tech 23:359-365, 2010 
21. Omeis I, Bekelis K, Gregory A, McGirt M, Sciubba D, Bydon A, et al: The use of expandable cages in patients undergoing multilevel corpectomies for metastatic tumors in the cervical spine. Orthopedics 33:87-92, 2010

22. Puttlitz CM, Harms J, Xu Z, Deviren V, Melcher RP: A biomechanical analysis of C2 corpectomy constructs. Spine J 7:210-215, 2007

23. Rhines LD, Fourney DR, Siadati A, Suk I, Gokaslan ZL: En bloc resection of multilevel cervical chordoma with C-2 involvement. Case report and description of operative technique. J Neurosurg Spine 2:199-205, 2005

24. Scheer JK, Tang J, Eguizabal J, Farin A, Buckley JM, Deviren V, et al: Optimal reconstruction technique after C-2 corpectomy and spondylectomy: a biomechanical analysis. J Neurosurg Spine 12:517-524, 2010

25. Sciubba DM, Chi JH, Rhines LD, Gokaslan ZL: Chordoma of the spinal column. Neurosurg Clin N Am 19:5-15, 2008

26. Štulík J, Kozák J, Šebesta P, Vyskočil T, Kryl J, Klezl Z: Total spondylectomy of C2: report of three cases and review of the literature. J Spinal Disord Tech 23:e53-e58, 2010

27. Suchomel P, Buchvald P, Barsa P, Froehlich R, Choutka O, Krejzar Z, et al: Single-stage total C-2 intralesional spondylectomy for chordoma with three-column reconstruction. Technical note. J Neurosurg Spine 6:611-618, 2007

28. Tomita K, Kawahara N, Baba H, Tsuchiya H, Fujita T, Toribatake Y: Total en bloc spondylectomy. A new surgical technique for primary malignant vertebral tumors. Spine (Phila Pa 1976) 22:324-333, 1997

29. Tomita K, Kawahara N, Murakami H, Demura S: Total en bloc spondylectomy for spinal tumors: improvement of the technique and its associated basic background. J Orthop Sci 11:3-12, 2006

30. Wang VY, Deviren V, Ames CP: Reconstruction of C-1 lateral mass with titanium mesh cage after resection of an aneurysmal bone cyst of the atlas. J Neurosurg Spine 10:117-121, 2009

31. White AA III, Panjabi MM (eds): Kinematics of the spine, in: Clinical Biomechanics of the Spine, ed 2. Philadephia: Lippincott Williams \& Wilkins, 1990, pp 85-126

32. Wolfla CE: Anatomical, biomechanical, and practical considerations in posterior occipitocervical instrumentation. Spine J 6 (6 Suppl):225S-232S, 2006

33. Yamazaki T, McLoughlin GS, Patel S, Rhines LD, Fourney DR: Feasibility and safety of en bloc resection for primary spine tumors: a systematic review by the Spine Oncology Study Group. Spine (Phila Pa 1976) 34 (22 Suppl):S31-S38, 2009

\section{Author Contributions}

Conception and design: Clarke, Gokaslan, Wolinsky. Acquisition of data: Clarke, Zadnik, Groves. Analysis and interpretation of data: Clarke, Zadnik, Groves. Drafting the article: Clarke, Zadnik, Groves, Sciubba, Wolinsky. Critically revising the article: all authors. Reviewed submitted version of manuscript: all authors. Approved the final version of the manuscript on behalf of all authors: Clarke. Statistical analysis: Clarke, Wolinsky. Administrative/technical/material support: Clarke. Study supervision: Clarke.

\section{Correspondence}

Michelle J. Clarke, Department of Neurosurgery, Go8S, Mayo Clinic, 200 First St. SW, Rochester, MN 55902. email: clarke. michelle@mayo.edu. 